\title{
Topics on global analysis of manifolds and representation theory of reductive groups
}

\author{
Toshiyuki Kobayashi \\ Graduate School of Mathematical Sciences, The University of Tokyo, \\ and Kavli IPMU (WPI)
}

\begin{abstract}
Geometric symmetry induces symmetries of function spaces, and the latter yields a clue to global analysis via representation theory. In this note we summarize recent developments on the general theory about how geometric conditions affect representation theoretic properties on function spaces, with focus on multiplicities and spectrum.

Mathematic Subject Classification (2020): Primary 22E46; Secondary $43 \mathrm{~A} 85,22 \mathrm{~F} 30$

\section{1 "Grip strength" of representations on global analysis - geometric criterion for finite- ness of multiplicities}

To which extent, does representation theory provide a useful information for global analysis on manifolds?

As a guiding principle, we begin with the following perspective ([14]).

Basic Problem 1.1 ("Grip strength" of representations). Support that a Lie group $G$ acts on $X$. Can the space of functions on $X$ be "sufficiently controlled" by the representation theory of $G$ ?

The vague words, "sufficiently controlled", or conversely, "uncontrollable", need to be formulated as mathematics. Let us observe what may happen in the general setting of infinite-dimensional representations of Lie groups $G$. 
Observation 1.2. For an infinite-dimensional $G$-module $V$, there may exist infinitely many different irreducible subrepresentations. Also, the multiplicity of each irreducible representation can range from finite to infinite.

When confronting such a general situation, one focuses on the principle:

- even though there are infinitely many (sometimes uncountably many) different irreducible representations, the group action can distinguish the different parts;

- the group action cannot distinguish the parts where the same irreducible representations occur with multiplicities.

This observation suggests us to think of the multiplicity of irreducible representations as an obstruction of "grip strength of a group". For each irreducible representation $\Pi$ of a group $G$, we define the multiplicity of $\Pi$ in the regular representation $C^{\infty}(X)$ by

$$
\operatorname{dim}_{\mathbb{C}} \operatorname{Hom}_{G}\left(\Pi, C^{\infty}(X)\right) \in \mathbb{N} \cup\{\infty\} .
$$

The case $\operatorname{dim}_{\mathbb{C}} \operatorname{Hom}_{G}\left(\Pi, C^{\infty}(X)\right)=1$ (multiplicity-one) provides a strong "grip strength" of representation theory on global analysis, which may be illuminated by the following example:

Example 1.3. Let $X$ be a manifold, $D_{1}, \cdots, D_{k}$ differential operators on $X$, and $G$ the group of diffeomorphisms $T$ of $X$ such that $T \circ D_{j}=D_{j} \circ T$ for all $j=1, \cdots, k$. Then the space of solutions $f$ to the differential equations on $X$ :

$$
D_{j} f=\lambda_{j} f \quad \text { for } 1 \leq j \leq k
$$

forms a $G$-module (possibly, zero) for any $\lambda_{1}, \cdots, \lambda_{k} \in \mathbb{C}$. The group $G$ becomes a Lie group if $\left\{D_{1}, \cdots, D_{k}\right\}$ contains the Laplacian when $X$ is a Riemannian manifold (or more generally a pseudo-Riemannian manifold). Assume now that the multiplicity of an irreducible representation $\Pi$ of $G$ in $C^{\infty}(X)$ is one. Then any function belonging to the image of a $G$ homomorphism from $\Pi$ to $C^{\infty}(X)$ satisfies a system of differential equations (1.2) for some $\lambda_{1}, \cdots, \lambda_{k} \in \mathbb{C}$ by Schur's lemma.

We formalise Basic Problem 1.1 as follows. 
Problem 1.4 (Grip strength of representations on global analysis). Let $X$ be a manifold on which a Lie group $G$ acts. Consider the regular representation of $G$ on $C^{\infty}(X)$ by

$$
C^{\infty}(X) \ni f(x) \mapsto f\left(g^{-1} \cdot x\right) \in C^{\infty}(X) \text { for } g \in G .
$$

(1) Find a necessary and sufficient on the pair $(G, X)$ for which the multiplicity (1.1) of every irreducible representation $\Pi$ of $G$ in the regular representation $C^{\infty}(X)$ is finite.

(2) Determine a condition on the pair $(G, X)$ for which the multiplicity is uniformly bounded with respect to all irreducible representations $\Pi$.

A solution to Problem 1.4 will single out a nice setting of $(G, H)$ in which we could expect a detailed study of global analysis on the homogeneous manifold $X=G / H$ by using representation theory of $G$. The multiplicity may depend on the irreducible representations $\Pi$ in (1), and thus we may think that the group $G$ has "stronger grip power" in (2) than in (1). We may also consider a multiplicity-free case:

(3) Determine a condition on the pair $(G, X)$ for which the multiplicity (1.1) is either 0 or 1 for any irreducible representation $\Pi$ of $G$.

Clearly, (3) is stronger than (2), however, we do not discuss (3) here.

Problem 1.4 is settled in Kobayashi-Oshima [17] for homogeneous spaces $X$ of reductive Lie groups $G$. To state the necessary and sufficient condition, we recall some notions from the theory of transformation groups. The following terminology was introduced in [11].

Definition 1.5 (Real sphericity). Suppose that a reductive Lie group $G$ acts continuously on a connected real manifold $X$. We say $X$ is a real spherical if a minimal parabolic subgroup of $G$ has an open orbit in $X$.

As is seen in Example 1.6 below, the classical notion of spherical varieties is a special case of real sphericity because a minimal parabolic subgroup of a complex reductive group $G_{\mathbb{C}}$ is nothing but a Borel subgroup.

Example 1.6 (Spherical variety). Suppose that $X_{\mathbb{C}}$ is a connected complex manifold and that a complex reductive Lie group $G_{\mathbb{C}}$ acts biholomorphically on $X_{\mathbb{C}}$. Then $X_{\mathbb{C}}$ is called a spherical variety of $G_{\mathbb{C}}$, if a Borel subgroup of $G_{\mathbb{C}}$ has an open orbit in $X_{\mathbb{C}}$. Spherical varieties have been extensively studied in algebraic geometry, geometric representation theory, and number theory. 
Here are some further examples.

Example 1.7. Let $X$ be a homogeneous space of a reductive Lie group $G$ and $X_{\mathbb{C}}$ its complexification.

(1) The following basic implications hold (Aomoto, Wolf, and KobayashiOshima).

\author{
$X$ is a symmetric space \\ $\Downarrow$ Aomoto, Wolf \\ $X_{\mathbb{C}}$ is a spherical variety \\ $\Downarrow$ Kobayashi-Oshima [17, Prop. 4.3] \\ $X$ is a real spherical variety \\ 介 obvious \\ $G$ is compact.
}

(2) When $X$ admits a $G$-invariant Riemannian structure, the following are equivalent (see Vinberg [24], Wolf [25]):

$X_{\mathbb{C}}$ is spherical

$\Longleftrightarrow X$ is weakly symmetric in the sense of Selberg

$\Longleftrightarrow X$ is a commutative space.

(3) The classification of irreducible symmetric spaces was accomplished by Berger [3] at the level of Lie algebras.

(4) The classification theory of spherical varieties $X_{\mathbb{C}}$ has been developed by Krämer, Brion, Mikityuk, and Yakimova.

(5) The triple space $(G \times G \times G) / \operatorname{diag} G$ is not a symmetric space. It is real spherical if and only if $G$ is locally a direct product of compact Lie groups and $S O(n, 1)$, see [11]. This geometric result implies a finiteness criterion of multiplicities for the tensor product of two infinite-dimensional irreducible representations ([11], [12, Cor. 4.2]). The triple space is considered as a special case of the homogeneous space $(\widetilde{G} \times G) / \operatorname{diag} G$ for a pair of groups $\widetilde{G} \supset G$. More generally, the classification of real spherical manifolds $(\widetilde{G} \times$ $G) / \operatorname{diag} G$ was accomplished in [16] when $(\widetilde{G}, G)$ are irreducible symmetric pairs in connection to the branching problem for $\widetilde{G} \downarrow G$, see [12]. 
(6) Let $N$ be a maximal unipotent subgroup of a real reductive Lie group $G$. Then $G / N$ is real spherical, as is easily seen from the Bruhat decomposition. Moreover, the following equivalence holds:

$$
G_{\mathbb{C}} / N_{\mathbb{C}} \text { is spherical } \Longleftrightarrow G \text { is quasi split. }
$$

This is related to the fact that the theory of Whittaker models (e.g. KostantLynch, H. Matumoto) yields stronger consequences when $G$ is assumed to be quasi split, see Remark 1.13 below.

We denote by $\operatorname{Irr}(G)$ the set of equivalence classes of irreducible admissible smooth representations of $G$. We do not assume unitarity for here. The solutions of Problem 1.4, which is a reformalisation of Basic Problem 1.1, are given by the following two theorems.

Theorem 1.8 (Criterion for finiteness of multiplicity [17]). Let $G$ be a reductive Lie group and $H$ a reductive algebraic subgroup of $G$, and set $X=G / H$. Then the following two conditions on the pair $(G, H)$ are equivalent.

(i) (representation theory) $\operatorname{dim}_{\mathbb{C}} \operatorname{Hom}_{G}\left(\Pi, C^{\infty}(X)\right)<\infty\left({ }^{\forall} \Pi \in \operatorname{Irr}(G)\right)$.

(ii) (geometry) $X$ is a real spherical variety.

In [17], the proof of the implication (ii) $\Rightarrow$ (i) uses (hyperfunction-valued) boundary maps for a system of partial differential equations with regular singularities, whereas that of the implication (i) $\Rightarrow$ (ii) is based on a generalization of the Poisson transform. This proof gives not only the equivalence of (i) and (ii) in Theorem 1.8 but also some estimates of the multiplicity from above and below. In turn, these estimates bring us to the following geometric criterion of the uniform boundedness of multiplicity.

Theorem 1.9 (Criterion for uniform boudedness of multiplicity [17]). Let $G$ be a reductive Lie group and $H$ a reductive algebraic subgroup of $G$, and set $X=G / H$. Then the following three conditions on the pair $(G, H)$ are equivalent.

(i) (representation theory) There exists a constant $C$ such that

$$
\operatorname{dim}_{\mathbb{C}} \operatorname{Hom}_{G}\left(\Pi, C^{\infty}(X)\right) \leq C \quad\left({ }^{\forall} \Pi \in \operatorname{Irr}(G)\right) .
$$

(ii) (complex geometry) The complexification $X_{\mathbb{C}}$ of $X$ is a spherical variety of $G_{\mathbb{C}}$.

(iii) (ring theory) The ring of $G$-invariant differential operators on $X$ is commutative. 
Remark 1.10. The equivalence (ii) $\Leftrightarrow$ (iii) in Theorem 1.9 is classical, see e.g., [24], and the main part here is to characterize the representation theoretic property (i) by means of conditions in other disciplines.

Remark 1.11. In general, the constant $C$ in (i) cannot be taken to be 1 when $H$ is noncompact.

Remark 1.12. Theorem 1.9 includes the discovery that the property of "uniform boundedness of multiplicity" is determined only by the complexification $\left(G_{\mathbb{C}}, X_{\mathbb{C}}\right)$ and is independent of a real form $(G, X)$. It is expected that this kind of statements could be generalized for reductive algebraic groups over non-archimedean local fields. Recently, Sakellaridis-Venkatesh [20] has obtained some affirmative results in this direction.

Remark 1.13. Theorems 1.8 and 1.9 give solutions to Problem 1.4 (1) and (2), respectively. More generally, these theorems hold not only for the space $C^{\infty}(X)$ of functions but also for the space of distributions and the space of sections of an equivariant vector bundle. Furthermore, a generalization dropping the assumption that the subgroup $H$ is reductive also holds, see [17, Thm. A, Thm. B] for precise formulation. For instance, the theory of the Whittaker model considers the case where $H$ is a maximal unipotent subgroup, see also Example 1.7 (5). Even for such a case a generalization of Theorems 1.8 and 1.9 can be applied.

Remark 1.14. We may also consider parabolic subgroups $Q$ instead of a minimal parabolic subgroup. In this case, we can also consider "generalized Poisson transform", and extend the implication (i) $\Rightarrow$ (ii) in Theorem 1.8, see [12, Cor. 6.8] for a precise formulation. On the other hand, an opposite implication (ii) $\Rightarrow$ (i) for parabolic subgroups $Q$ is not always true, see Tauchi [22].

Theorems 1.8 and 1.9 suggest nice settings of global analysis in which the "grip strength" of representation theory is "strong". The Whittaker model and the analysis on semisimple symmetric spaces may be thought of in this framework as was seen in (6) and (1), respectively, of Example 1.7. As yet another set of problems, let us discuss briefly the restriction of representations to subgroups (branching problems).

In the spirit of "grip strength" (Basic Problem 1.1), we may ask "grip strength of a subgroup" on an irreducible representation of a larger group as follows: 
Basic Problem 1.15 (Grip strength in branching problem). Let $\Pi$ be an irreducible representation of a group $G$. We regard $\Pi$ as a representation of a subgroup $G^{\prime}$ by restriction, and consider how many times another irreducible representation $\pi$ of $G^{\prime}$ occurs in the restriction $\left.\Pi\right|_{G^{\prime}}$ :

(1) When is the multiplicity of every irreducible representation $\pi$ of $G^{\prime}$ occurring in the restriction $\left.\Pi\right|_{G^{\prime}}$ finite?

(2) When is the multiplicity of irreducible representation $\pi$ of $G^{\prime}$ occurring in the restriction $\left.\Pi\right|_{G^{\prime}}$ uniformly bounded?

To be precise, we need to clarify what "occur" means, e.g., as a submodule, as a quotient, or as a support of the direct integral (2.1) of the unitary representation, etc. Furthermore, since our concern is with infinitedimensional irreducible representations, the definition of "multiplicity" depends also on the topology of the representation spaces of $\Pi$ of $G$ and $\pi$ of $G^{\prime}$. Typical definitions of multiplicities include:

$$
\begin{aligned}
& \operatorname{dim} \operatorname{Hom}_{G^{\prime}}\left(\left.\Pi^{\infty}\right|_{G^{\prime}}, \pi^{\infty}\right), \\
& \operatorname{dim} \operatorname{Hom}_{G^{\prime}}\left(\left.\Pi\right|_{G^{\prime}}, \pi\right), \\
& \operatorname{dim} \operatorname{Hom}_{G^{\prime}}\left(\pi,\left.\Pi\right|_{G^{\prime}}\right), \\
& \operatorname{dim} \operatorname{Hom}_{\mathfrak{g}^{\prime}, K^{\prime}}\left(\pi_{K^{\prime}}, \Pi_{K}\right) .
\end{aligned}
$$

Here $\Pi^{\infty}, \pi^{\infty}$ stand for smooth representations, whereas $\pi_{K^{\prime}}$ and $\Pi_{K}$ stand for the underlying $\left(\mathfrak{g}^{\prime}, K^{\prime}\right)$-modules and $(\mathfrak{g}, K)$-modules. If $\Pi$ and $\pi$ are both unitary representations, then the quantities (1.4) and (1.5) coincide. If (1.6) $\neq 0$ in addition, then all the quantities (1.3)-(1.6) coincide. In general the multiplicity (1.6) often vanishes, and its criterion is given in [9, 10].

Concerning the multiplicity (1.3), see [12], 21] and references therein for the general theory, in particular, for a geometric necessary and sufficient condition on the pair $\left(G, G^{\prime}\right)$ such that (1) (or more strongly (2)) of Basic Problem 1.15 is always fulfilled. When the triple (ח, $\left.G, G^{\prime}\right)$ satisfies finiteness (or more strongly, uniform boundedness) of the multiplicity in Basic Problem 1.15, we could expect a detailed study of the restriction $\left.\Pi\right|_{G^{\prime}}$, see [13], for further "programs" of branching problems of reductive groups, such as the construction of "symmetry breaking operators" and "holographic operators" in concrete settings [15, 18, 19]. 


\section{Spectrum of the regular representation $L^{2}(X)$ - a geometric criterion for temperdness}

In the previous section, we focused on "multiplicity" from the perspective of "grip strength" of a group on a function space and proposed (real) spherical varieties as "a nice framework for detailed study of global analysis". On the other hand, even in a case in which the "grip strength" of representation theory is "weak", we may still expect to analyze the space of functions on $X$ from representation theory in a "coarse standpoint". In this section, including non-spherical cases, let us focus on the support of the Plancherel measure and consider the following problem.

Suppose that a Lie group $G$ acts on a manifold $X$ with a Radon measure $\mu$ and that $G$ leaves the measure invariant so that $G$ acts naturally on the Hilbert space $L^{2}(X) \equiv L^{2}(X, d \mu)$ as a unitary representation.

Basic Problem 2.1 (Tempered space [1]). Find a necessary and sufficient condition on a pair $(G, X)$ for which the regular representation $L^{2}(X)$ of $G$ is a tempered representation.

We recall the general definition of tempered representations.

Definition 2.2 (Tempered representation). A unitary representation $\pi$ of a locally compact group $G$ is called tempered if $\pi$ is weakly contained in $L^{2}(G)$, namely, if any matrix coefficient $G \ni g \mapsto(\pi(g) u, v) \in \mathbb{C}$ can be approximated by a sequence of linear combinations of matrix coefficients of the regular representation $L^{2}(G)$ on every compact set of $G$.

The classification of irreducible tempered representations of real reductive linear Lie groups $G$ was accomplished by Knapp-Zuckerman [5]. In contrast to the long-standing problem of the classification of the unitary dual $\widehat{G}$, irreducible tempered representations form a subset of $\widehat{G}$ that is fairly wellunderstood. Loosely speaking, from the orbit philosophy due to KirillovKostant-Duflo, irreducible tempered representations are supposed to be obtained as a "geometric quantization" of regular semisimple coadjoint orbits, see e.g., 4, 8].

Tempered representations are unitary representations by definition, however, the classification theory of Knapp-Zuckerman played also a crucial role in the Langlands classification of irreducible admissible representations (without asking if they are unitarizable or not) of real reductive Lie groups. 
The general theory of Mautner-Teleman tells that any unitary representation $\Pi$ of a locally compact group $G$ can be decomposed into the direct integral of irreducible unitary representations:

$$
\Pi \simeq \int^{\oplus} \pi_{\lambda} d \mu(\lambda) .
$$

Then the following equivalence (i) $\Leftrightarrow$ (ii) holds ([1, Rem. 2.6]):

(i) $\Pi$ is tempered;

(ii) irreducible representation $\pi_{\lambda}$ is tempered for a.e. $\lambda$ with respect to the measure $\mu$.

The irreducible decomposition of the regular representation of $G$ on $L^{2}(X)$ is called the Plancherel-type theorem for $X$. Thus, if the Plancherel formula is "known", then we should be able to answer Basic Problem 2.1] in principle. However, things are not so easy:

Observation 2.3. The Plancherel-type theorem for semisimple symmetric spaces $G / H$ was proved by T. Oshima, P. Delorme, E. van den Ban, and H. Schlichtkrull (up to nonvanishing condition of discrete series representation with singular parameters). However, it seems that a necessary and sufficient condition on a symmetric pair $(G, H)$ for which $L^{2}(G / H)$ is tempered had not been found until the general theory [1 is established by a completely different approach. In fact, it is possible to show that temperedness of $L^{2}(G / H)$ implies a simple geometric condition that $(G / H)_{\mathrm{Am}}$ is dense in $G / H$ (see the second statement of Theorem 2.11) from their Planchereltype formula in the case where $G / H$ is a symmetric space, whereas there is a counterexample to the converse statement, as was found in [1]. If one employs the Plancherel-type formula in order to derive the right answer to Problem 2.1 for symmetric spaces $G / H$, one will need a precise (non-)vanishing condition on certain cohomologies (Zuckerman derived functor modules) with singular parameters, and such a condition is combinatorially complicated in many cases $([7,23])$.

Observation 2.4. More generally, when $X_{\mathbb{C}}$ is not necessarily a spherical variety of $G_{\mathbb{C}}$, as shown in Theorem 1.9 , the ring $\mathbb{D}_{G}(X)$ of $G$-invariant differential operators on $X$ is not commutative and so we cannot use effectively the existing method on non-commutative harmonic analysis based on an expansion of functions on $X$ into joint eigenfunctions with respect to the commutative ring $\mathbb{D}_{G}(X)$, cf. Example 1.3. 
As observed above, to tackle Basic Problem 2.1, one needs to develop a new method itself. As a new approach, Benoist and I utilised an idea of dynamical system rather than differential equations. We begin with some basic notion:

Definition 2.5 (Proper action). Suppose that a locally compact group $G$ acts continuously on a locally compact space $X$. This action is called proper if the map

$$
G \times X \rightarrow X \times X, \quad(g, x) \mapsto(x, g \cdot x)
$$

is proper, namely, if

$$
G_{S}:=\{s \in G: g S \cap S \neq \emptyset\}
$$

is compact for any compact subset $S$ of $X$.

If $G$ acts properly on $X$, then the stabilizer of any point $x \in X$ in $G$ is compact. On the other hand, if $H$ is a compact subgroup of $G$, then $L^{2}(G / H) \subset L^{2}(G)$ holds, hence the regular representation on $L^{2}(G / H)$ is tempered. The following can be readily drawn from this.

Example 2.6. If the action of a group $G$ on $X$ is proper (Definition 2.5), then the regular representation in $L^{2}(X)$ is tempered.

Therefore, in the study of Basic Problem 2.1, we focus on the nontrivial case that the action of $G$ on $X$ is not proper. Properness of the action is qualitative property, namely, there exists a compact subset $S$ of $X$ such that the set $G_{S}=\{g \in G: g S \cap S \neq \emptyset\}$ is noncompact. In order to shift it quantitatively, we consider the volume $\operatorname{vol}(g S \cap S)$. Viewed as a function on $G$,

$$
G \ni g \mapsto \operatorname{vol}(g S \cap S) \in \mathbb{R}
$$

is a continuous function of $g \in G$. Definition 2.5 tells that the $G$-action on $X$ is not proper if and only if the support of the function (2.2) is noncompact for some compact subset $S$ of $X$. Hence the "decay" of the function (2.2) at infinity may be considered as capturing quantitatively a "degree" of nonproperness of the action. By pursuing this idea, Basic Problem 2.1 is settled in Benoist-Kobayashi [1, 2] when $X$ is an algebraic $G$-variety for a reductive group $G$. To describe the solution, let us introduce a piecewise linear function associated to a finite-dimensional representation of a Lie algebra. 
Definition 2.7. For a representation $\sigma: \mathfrak{h} \rightarrow \operatorname{End}_{\mathbb{R}}(V)$ of a Lie algebra $\mathfrak{h}$ on a finite-dimensional real vector space $V$, we define a function $\rho_{V}$ on $\mathfrak{h}$ by

$$
\begin{aligned}
\rho_{V}: \mathfrak{h} \rightarrow \mathbb{R}, \quad Y & \mapsto \text { the sum of the absolute values of the real parts } \\
& \text { of the eigenvalues of } \sigma(Y) \text { on } V \otimes_{\mathbb{R}} \mathbb{C} .
\end{aligned}
$$

The function $\rho_{V}$ is uniquely determined by the restriction to a maximal abelian split subalgebra $\mathfrak{a}$ of $\mathfrak{h}$. Further, the restriction $\left.\rho_{V}\right|_{\mathfrak{a}}$ is a piecewise linear function on $\mathfrak{a}$, namely, there exist finitely many convex polyhedral cones which cover $\mathfrak{a}$ and on which $\rho_{V}$ is linear.

Example 2.8. When $(\sigma, V)$ is the adjoint representation $(\mathrm{ad}, \mathfrak{h})$, the restriction $\left.\rho_{\mathfrak{h}}\right|_{\mathfrak{a}}$ can be computed by using a root system. It coincides with twice the usual " $\rho$ " in the dominant Weyl chamber.

With this notation, one can describe a necessary and sufficient condition for Basic Problem 2.1.

Theorem 2.9 (Criterion for temperedness of $L^{2}(X)$, [2]). Let $G$ be a reductive Lie group and $H$ a connected closed subgroup of $G$. We denote by $\mathfrak{g}$ and $\mathfrak{h}$ the Lie algebras of $G$ and $H$, respectively. Then the following two conditions on a pair $(G, H)$ are equivalent.

(i) (global analysis) The regular representation $L^{2}(G / H)$ is tempered.

(ii) (combinatorial geometry) $\rho_{\mathfrak{h}} \leq \rho_{\mathfrak{g} / \mathfrak{h}}$.

Remark 2.10. If $G$ is an algebraic group acting on an algebraic variety $X$, then, even when $X$ is not a homogeneous space of $G$, one can give an answer to Basic Problem 2.1 by applying Theorem 2.9 to generic $G$-orbits ([2]).

Theorem 2.9 was proved in [1] in the special case where both $G$ and $H$ are real algebraic reductive groups. In this case, the following theorem also holds:

Theorem $2.11([1])$. Let $G \supset H$ be a pair of real algebraic reductive Lie group. We set

$(G / H)_{\mathrm{Am}}:=\{x \in G / H$ : the stabilizer of $x$ in $H$ is amenable $\}$

$(G / H)_{\mathrm{Ab}}:=\{x \in G / H$ : the stabilizer of $x$ in $H$ is abelian $\}$. 
Then the following implications hold.

$$
\begin{array}{lc}
\text { geometry } & (G / H)_{\mathrm{Ab}} \text { is dense in } G / H \\
\Downarrow & \Downarrow \\
\text { representation } & L^{2}(G / H) \text { is tempered } \\
\Downarrow & \Downarrow \\
\text { geometry } & (G / H)_{\mathrm{Am}} \text { is dense in } G / H .
\end{array}
$$

Since a complex Lie group is amenable if and only if it is abelian, Theorem 2.11 implies the following:

Corollary 2.12. The following conditions on a pair of complex reductive Lie groups $(G, H)$ are equivalent:

(i) $L^{2}(G / H)$ is tempered.

(ii) $(G / H)_{\mathrm{Ab}}$ is dense in $G / H$.

Acknowledgements: The author was partially supported by Grant-inAid for Scientific Research (A) (18H03669), Japan Society for the Promotion of Science. He also would like to thank Professor Vladimir Dobrev for his warm hospitality.

\section{References}

[1] Y. Benoist, T. Kobayashi, Temperedness of reductive homogeneous spaces, J. Eur. Math. Soc., 17 (2015), pp. 3015-3036.

[2] Y. Benoist, T. Kobayashi, Tempered homogeneous spaces, to appear in Margulis Festschrift, available at arXiv:1706.10131.

[3] M. Berger, Les espaces symétriques non compacts, Ann. Sci. École Norm. Sup. 74 (1957), 85-177.

[4] A. A. Kirillov, Lectures on the Orbit Method, Grad. Stud. Math., 64 Amer. Math. Soc. 2004.

[5] A. W. Knapp and G. J. Zuckerman, Classification of irreducible tempered representations of semisimple groups, Ann. of Math. (2) 116 (1982), no. 2, 389-455; II. ibid., 457-501. 
[6] T. Kobayashi, Proper action on a homogeneous space of reductive type, Math. Ann. 285 (1989), pp. 249-263.

[7] T. Kobayashi, Singular unitary representations and discrete series for indefinite Stiefel manifolds $U(p, q ; \mathbb{F}) / U(p-m, q ; \mathbb{F})$, Mem. Amer. Math. Soc. 95, no. 462, vi+106 pp. (1992).

[8] T. Kobayashi, Harmonic analysis on homogeneous manifolds of reductive type and unitary representation theory, Translations, Series II, Selected Papers on Harmonic Analysis, Groups, and Invariants, 183 (1998), Amer. Math. Soc., pp. 1-31 ; translated from Sugaku, 46 (1994), pp. 124-143.

[9] T. Kobayashi, Discrete decomposability of the restriction of $A_{\mathfrak{q}}(\lambda)$ with respect to reductive subgroups II - micro-local analysis and asymptotic K-support, Ann. of Math., 147 (1998), pp. 709-729.

[10] T. Kobayashi, Discrete decomposability of the restriction of $A_{\mathfrak{q}}(\lambda)$ with respect to reductive subgroups III-restriction of Harish-Chandra modules and associated varieties, Invent. Math., 131 (1998), pp. 229-256.

[11] T. Kobayashi, Introduction to harmonic analysis on real spherical homogeneous spaces, Proceedings of the 3rd Summer School on Number Theory "Homogeneous Spaces and Automorphic Forms" in Nagano (F. Sato, ed.), 1995, 22-41 (in Japanese).

[12] T. Kobayashi, Shintani functions, real spherical manifolds, and symmetry breaking operators, Dev. Math., 37 (2014), pp. 127-159.

[13] T. Kobayashi, A program for branching problems in the representation theory of real reductive groups, In: Representations of Lie Groups: In Honor of D. A. Vogan, Jr. on his 60th Birthday, Progr. Math., 312 pp. 277-322, Birkhäuser, 2015.

[14] T. Kobayashi, Recent advances in branching laws of representations, Sugaku (2019), 71 (4), pp. 388-416, English translation to appear from AMS.

[15] T. Kobayashi, T. Kubo, M. Pevzner, Conformal Symmetry Breaking Operators for Differential Forms on Spheres, Lecture Notes in Math., 2170 Springer, 2016, viii + 192 pages.

[16] T. Kobayashi, T. Matsuki, Classification of finite-multiplicity symmetric pairs, Transform. Groups 19 (2014), 457-493, Special issue in honor of Dynkin for his 90 th birthday. 
[17] T. Kobayashi, T. Oshima, Finite multiplicity theorems for induction and restriction, Adv. Math., 248 (2013), pp. 921-944.

[18] T. Kobayashi, B. Speh, Symmetry breaking for representations of rank one orthogonal groups, (2015), Mem. Amer. Math. Soc. 238 no.1126, 118 pages.

[19] T. Kobayashi, B. Speh, Symmetry Breaking for Representations of Rank One Orthogonal Groups, Part II, Lecture Notes in Math., 2234, Springer, 2018. xv+342 pages.

[20] Y. Sakellaridis and A. Venkatesh, Periods and Harmonic Analysis on Spherical Varieties, Asterisque, 396, Soc. Math. France, 2018.

[21] B. Sun, C.-B. Zhu, Multiplicity one theorems: the Archimedean case, Ann. of Math., 175 (2012), pp. 23-44.

[22] T. Tauchi, Dimension of the space of intertwining operators from degenerate principal series representations, Selecta Math. (N.S.) 24, (2018), pp. 3649-3662.

[23] P. E. Trapa, Annihilators and associated varieties of $A_{\mathfrak{q}}(\lambda)$ modules for $U(p, q)$, Compos. Math. 129, 1-45 (2001).

[24] E. B. Vinberg, Commutative homogeneous spaces and co-isotropic symplectic actions, Russian Math. Surveys, 56, (2001), pp. 1-60.

[25] J. A. Wolf, Harmonic Analysis on Commutative Spaces, Mathematical Surveys and Monographs, 142, Amer. Math. Soc. 2007, xvi+387 pages. 